\title{
RESPOSTA SEXUAL AUTORREFERIDA DA MULHER NA TERCEIRA IDADE
}

\author{
Ana Fátima Souza Melo de Andrade', Luana da Conceição Costa Cardoso², Taciana Silveira Passos³ \\ FACTORS THAT INTERFERE WITH THE SEXUAL RESPONSE OF WOMEN IN THE THIRD AGE
}

\section{FACTORES QUE INTERFIEREN CON LA RESPUESTA SEXUAL DE LAS MUJERES EN LA TERCERA EDAD}

\begin{abstract}
Resumo: Apesar do aumento da expectativa de vida, a sexualidade no idoso não tem recebido a devida importância da sociedade. Com o envelhecimento, o corpo passa por alterações fisiológicas, psicológicas e sociais que interferem na prática sexual. $O$ objetivo do presente estudo foi descrever autorrelato de mulheres idosas sobre aspectos que interferem na resposta sexual. Trata-se de um estudo descritivo com amostra de 65 idosas cadastradas em um Centro de Referência da Assistência Social (CRAS) do município de Aracaju (SE). Foi utilizado um questionário semiestruturado. $O$ teste $X^{2}$ de Pearson foi utilizado para associação das variáveis. Considerou-se significativo o valor $p \leq 0,05$. Os resultados apontaram que $52,54 \%$ das entrevistadas eram viúvas e $61 \%$ eram sexualmente inativas. $O$ fato de ser viúva $(p=0,007)$, baixa escolaridade $(p=0,00 \mathrm{I})$ e dormir em quarto compartilhado $(p=0,006)$ esteve associado à inatividade sexual. As causas de inatividade mais relatadas foram a falta de um parceiro $(25 \%)$ e de privacidade (20\%). A maioria das mulheres sexualmente ativas mantinham relações por prazer $(86,95 \%)$. A idade não teve associação significativa com a atividade sexual. A crença de aceitação dos filhos esteve associada ao interesse das idosas em relacionar-se amorosamente após ficar viúva ou se divorciar $(p=0,003)$. Dessa forma, conclui-se que não foi a idade que interferiu nas relações afetivas e sexuais das idosas entrevistadas, e sim fatores sociais associados ao envelhecimento. Um dos desafios da área da saúde é quebrar os mitos e os preconceitos na sociedade e preparar futuros idosos para uma senescência mais saudável.
\end{abstract}

Palavras-Chave: Sexualidade. Envelhecimento. Alterações.

Abstract: Despite the increase in life expectancy, sexuality in the elderly has not received due importance from society. With aging, the body goes through physiological, psychological, and social changes that interfere with sexual practice. The aim of the present study was to describe self-reporting by elderly women about aspects that interfere with sexual response. This is a descriptive study with a sample of 65 elderly women registered at a Social Assistance Reference Center (CRAS) in the municipality of Aracaju-SE. A semi-structured questionnaire was used. Pearson's $\mathrm{X}^{2}$ test was used to associate the variables. A value of $p \leq 0.05$ was considered significant. The results showed that $52.54 \%$ of the interviewees were widows and $61 \%$ were sexually inactive. The fact of being a widow $(p=0.007)$, low education $(p=0.00 \mathrm{I})$ and sleeping in a shared room $(p=0.006)$ was associated with sexual inactivity. The most reported causes of inactivity were the lack of a partner $(25 \%)$ and privacy $(20 \%)$. Most sexually active women had sex for pleasure (86.95\%). Age had no significant association with sexual activity. The belief in the acceptance of children was associated with the interest of the elderly women in loving relationships after becoming a widow or divorcing $(p=0.003)$. Thus, it is concluded that it was not age that interfered in the affective and sexual relationships of the elderly women interviewed, but social factors associated with aging. One of the challenges in the health field is to break the myths and prejudices in society and prepare future elderly people for healthier senescence.

Keywords: Sexuality. Aging. Changes.

Resumen: A pesar del aumento en la esperanza de vida, la sexualidad en los ancianos no ha recibido la debida importancia de la sociedad. Con el envejecimiento, el cuerpo pasa por cambios fisiológicos, psicológicos y sociales que interfieren con la práctica sexual. El objetivo del presente estudio fue describir el autoinforme de las mujeres mayores

\footnotetext{
I Centro Universitário Estácio de Sergipe. E-mail: anafatimamelo@hotmail.com

2 Universidade Tiradentes. E-mail: luanacardoso.ccc@gmail.com

3 Universidade Tiradentes. E-mail: tacianasilveirapassos@gmail.com
} 
20 Revista Brasileira de

Sexualidade Humana

DOI: https://doi.org/10.35919/rbsh.v3Iil.293

sobre aspectos que interfieren con la respuesta sexual. Este es un estudio descriptivo con una muestra de 65 mujeres mayores registradas en un Centro de Referencia de Asistencia Social (CRAS) en el municipio de Aracaju-SE. Se utilizó un cuestionario semiestructurado. Se utilizó la prueba $X^{2}$ de Pearson para asociar las variables. Un valor de $p \leq 0.05$ fue considerado como significativo. Los resultados mostraron que el $52.54 \%$ de los entrevistados eran viudas y el $61 \%$ eran sexualmente inactivos. El hecho de ser viuda $(p=0.007)$, baja educación $(p=0.00 \mathrm{I})$ y dormir en una habitación compartida $(p=0.006)$ se asoció con la inactividad sexual. Las causas más reportadas de inactividad fueron la falta de pareja (25\%) y la privacidad (20\%). La mayoría de las mujeres sexualmente activas tenían relaciones sexuales por placer (86.95\%). La edad no tuvo asociación significativa con la actividad sexual. La creencia en la aceptación de los niños se asoció con el interés de las mujeres mayores en relacionarse amorosamente después de convertirse en viuda o divorciarse $(p=0.003)$. Por lo tanto, se concluye que no fue la edad lo que interfirió en las relaciones afectivas y sexuales de las mujeres mayores entrevistadas, sino los factores sociales asociados con el envejecimiento. Uno de los desafíos en el campo de la salud es romper los mitos y prejuicios en la sociedad y preparar a las futuras personas mayores para una senescencia más saludable.

Palabras clave: Sexualidad. Envejecimiento Cambios.

\section{Introdução}

A Organização Mundial da Saúde (OMS) define a população idosa como aquela a partir dos 60 anos de idade, limite válido para os países em desenvolvimento e, nos desenvolvidos, considera-se a partir de 65 anos (WORLD HEALTH ORGANIZATION, 2005). O enveIhecimento e crescimento dessa população são reflexos das mudanças positivas da qualidade de vida, como melhores condições de higiene, moradia, alimentação e controle das enfermidades. A consequência disso é a conquista da longevidade, que tem causado interesse crescente nos diversos campos do conhecimento (CAMPOS; FERREIRA; VARGAS, 20I5; SOUSA et al., 20I9).

Apesar do aumento da expectativa de vida, a sexualidade no idoso não tem recebido a devida importância da sociedade, visto que a prática sexual se torna extinta com o passar dos anos (EVANGELISTA et al., 2019). De acordo com Santos e demais autores (2017), a falta de uma educação sexual adequada, associada às repressões constantes na fase de descobrimento da sexualidade, levam a problemas de saúde pública.

A OMS define sexualidade como "um aspecto central do ser humano ao longo da vida que abrange sexo, identidades e papéis de gênero, orientação sexual, erotismo, prazer, intimidade e reprodução". Além disso, sofre influência da articulação de fatores biológicos, psicológicos, sociais, econômicos, políticos, culturais, legais, históricos, religiosos e espirituais (WORLD HEALTH ORGANIZATION, 2006).

Segundo Souza (20I6), a sexualidade nem sempre é tratada com abertura, pois se refere às experiências pessoais extremamente íntimas, especialmente quando tratamos sobre sexo na terceira idade. Esse é de difícil compreensão por parte das sociedades existentes, até mesmo para os jovens, agravando-se ainda mais no caso dos idosos, o que dificulta a elaboração de protocolos de assistência às pessoas idosas com disfunções sexuais.

De modo geral, a crença de que o avançar da idade e o declinar da prática sexual estejam diretamente ligados tem sido responsável para que não se dê a devida atenção à sexualidade, que é uma das atividades mais associadas à qualidade de vida. Acredita-se que através do esclarecimento das informações distorcidas que se propagam acerca da sexualidade, poderá haver a diminuição das crenças e tabus sobre um assunto repleto de preconceitos (FREIRE et al., 20I7; UCHÔA et al., 2016).

A prática sexual nessa faixa etária requer uma atenção especial, visto que no processo de envelhecimento ocorrem diversas transformações orgânicas, físicas e psicológicas, que também se interligam com a sexualidade. $O$ envelhecimento saudável e a qualidade de vida requerem uma compreensão clara de um conjunto de aspectos que contempla o cotidiano da pessoa idosa, identificar os fatores inerentes à sexualidade na terceira idade faz parte desse processo, trazendo subsídios para um melhor entendimento desse contexto dentro da sociedade (ARAÚJO; ZAZULA, 20I5; ARAÚJO et al., 2017).

O presente trabalho emerge com significativa relevância, dado o fato de que visa tratar do papel da sexualidade, enquanto fator positivo para a qualidade de vida, sendo necessária abordagem desse assunto por sabermos dos preconceitos existentes na sociedade, aliados à falta de informação, que reforçam os mitos relativos à velhice assexuada. Dessa forma, o estudo tem como objetivo geral descrever autorrelato de mulheres idosas sobre aspectos que interferem na resposta sexual.

\section{Material e métodos}

Trata-se de uma pesquisa de caráter descritivo e abordagem quantitativa. Realizada durante $\circ$ mês de 
novembro de 2012, com uma amostra composta de 65 mulheres idosas, cadastradas em um Centro de Referência de Assistência Social (CRAS) do $4^{\circ}$ distrito da saúde, localizado no município de Aracaju (SE).

Foram incluídas todas as mulheres a partir dos 60 anos de idade, cadastradas no Programa de Atenção Integral ao Idoso (PAPI), que aceitaram responder ao formulário; e excluídas aquelas com menos de 60 anos de idade, as que nunca tiveram relação sexual e as que se recusaram a participar da pesquisa.

O instrumento utilizado para coleta de dados foi um formulário semiestruturado, contendo 17 questões relacionadas a aspectos sociodemográficos e à vida sexual da mulher. O referido questionário passou por um processo de validação facial (SÁNCHEZ; ECHEVERRY, 2004) com cinco experts na área interdisciplinar relacionada aos temas da pesquisa.

O questionário foi aplicado por uma enfermeira em forma de entrevista individual, no espaço do CRAS, em datas preestabelecidas, no período compreendido entre 5 e 30 de novembro. Antes de aplicar o instrumento, foi apresentado às participantes da pesquisa, um Termo de Consentimento Livre e Esclarecido (TCLE), no qual foram orientadas sobre a relevância da pesquisa, 0 objetivo do estudo e confidencialidade dos dados. Após a concordância em participar da pesquisa, foi aplicado o formulário, e colocado dentro de um envelope fechado, sem identificação dos sujeitos envolvidos.

As variáveis analisadas nesse instrumento foram: os arranjos domiciliares, rendimento salarial, coabitação, as alterações fisiológicas de maior prevalência que acometem o sistema reprodutor feminino com o envelhecimento, o conhecimento sobre essas mudanças, a frequência da prática sexual, correlacionada aos fatores intrínsecos e extrínsecos.

Os dados foram tabulados através do Microsoft Excel. As variáveis categóricas foram apresentadas em números absolutos e porcentagem através de tabelas e gráficos. Posteriormente, o teste Qui-quadrado de Pearson foi utilizado para associação das proporções entre atividade sexual e características sociodemográficas. O nível de significância estatística utilizado foi de $5 \%$ ( $p$ menor ou igual 0,05 ).

A proposta foi aprovada pelo Comitê de Ética em Pesquisa (CEP) da Universidade Tiradentes (UNIT), através do parecer $n^{\circ} 0.509 .09 R$. Foi desenvolvido conforme os requisitos propostos pela Resolução $n^{\circ} 466 / 2012$ do Conselho Nacional de Saúde (CNS). Aquelas que concordaram em participar da pesquisa assinaram o TCLE.

\section{Resultados}

Em um total de 65 formulários, foram respondidos 59, sendo seis mulheres excluídas segundo os critérios preestabelecidos. Dos resultados obtidos, observa-se na Tabela I que em relação à idade, temos uma predominância da faixa etária de $60-70$ anos $(64,4 \%)$, seguida da faixa etária de $7 \mathrm{I}-80$ anos $(28,81 \%)$ e, por fim, acima de $8 \mathrm{I}$ anos (6,77\%). Em relação ao estado civil, $52,54 \%$ eram viúvas, $25,42 \%$, casadas, e $22,03 \%$, divorciadas.

Tabela I - Distribuição do perfil sociodemográfico das mulheres idosas do CRAS do município de Aracaju (SE) em 2012

\begin{tabular}{l|c|c}
\hline & $N$ & $\%$ \\
\hline Idade (anos) & 38 & \\
\hline $60-70$ & 17 & 64,4 \\
\hline $71-80$ & 04 & 28,8 \\
\hline 81 ou mais & \multicolumn{2}{c}{ Frequência } \\
\hline Estado civil & - & 2,8 \\
\hline Solteira & 15 & 25,4 \\
\hline Casada & 13 & 22,0 \\
\hline Divorciada & 31 & 52,6 \\
\hline Viúva & & \\
\hline Escolaridade & 10 & 16,9 \\
\hline Analfabeta & 38 & 64,4 \\
\hline Fundamental incompleto & 03 & 5,1 \\
\hline Fundamental completo & 01 & 1,7 \\
\hline Médio incompleto & & \\
\hline
\end{tabular}




\begin{tabular}{|c|c|c|}
\hline & \multicolumn{2}{|c|}{ Frequência } \\
\hline & $N$ & $\%$ \\
\hline Médio completo & 07 & 11,9 \\
\hline \multicolumn{3}{|l|}{ Renda familiar (salário mínimo) } \\
\hline Inferior ou igual a $0 \mathrm{I}$ salário & 22 & 37,3 \\
\hline Superior a 01 e inferior a 02 salários & 27 & 45,8 \\
\hline Superior a 02 salários & 10 & 16,9 \\
\hline \multicolumn{3}{|l|}{ Coabitação } \\
\hline Sozinha & 12 & 20,3 \\
\hline Filhos e/ou netos & 32 & 54,3 \\
\hline Marido e filhos & 15 & 25,4 \\
\hline \multicolumn{3}{|l|}{ Quarto compartilhado } \\
\hline Não compartilha & 36 & 61,0 \\
\hline Marido somente & 13 & 22,1 \\
\hline Parentes & 10 & 16,9 \\
\hline
\end{tabular}

Fonte: elaborada pelas autoras, 2012.

Quanto às condições socioeconômicas apresentadas na Tabela I, observou-se que 37,28\% têm renda inferior ou igual a um salário mínimo, 16,94\% eram analfabetas e $64,40 \%$ tinham ensino fundamental incompleto. Sobre condições de moradia, $20,33 \%$ moram sozinhas, $54,33 \%$ moram com filhos ou netos e $25,42 \%$ moram com marido e filhos. Em relação ao compartilhamento do cômodo onde dormem, $61,01 \%$ relataram que não compartilham o quarto, $22,03 \%$ dormem apenas com o marido e $16,94 \%$ dividem com parentes.

$\mathrm{Na}$ amostra do presente estudo, 23 mulheres (39\%) referiram ser sexualmente ativas e 36 inativas $(61 \%)$. O fato de ser viúva $(p=0,007)$, baixa escolaridade $(p=0,00 \mathrm{I})$ e dormir em quarto compartilhado $(p=0,006)$ esteve associado à inatividade sexual. $\mathrm{A}$ idade $\mathrm{e}$ a renda não tiveram associação estatisticamente significativa com a atividade sexual das idosas (Tabela 2).

Tabela 2 - Associação entre aspectos sociodemográficos e atividade sexual das mulheres idosas do CRAS do município de Aracaju (SE) em 2012

\begin{tabular}{|c|c|c|c|c|}
\hline & \multicolumn{3}{|c|}{ Frequência } & \multirow[t]{2}{*}{$\mathbf{p}$} \\
\hline & Ativas & Inativas & Total & \\
\hline & $\mathrm{N}(\%)$ & $N(\%)$ & $N(\%)$ & \\
\hline \multicolumn{4}{|l|}{ Idade (anos) } & 0,917 \\
\hline $60-70$ & $15(39,5)$ & $23(60,5)$ & $38(100,0)$ & \\
\hline$>70$ & $8(38,1)$ & $13(6 \mid, 9)$ & $21(100,0)$ & \\
\hline \multicolumn{4}{|l|}{ Estado civil } & 0,007 \\
\hline Casada/Divorciada & $16(57,1)$ & $12(42,9)$ & $28(100,0)$ & \\
\hline Viúva & $7(22,6)$ & $24(77,4)$ & $31(100,0)$ & \\
\hline \multicolumn{4}{|l|}{ Escolaridade } & 0,001 \\
\hline Analfabeta/Fundamental incompleto & $14(29,2)$ & $34(70,8)$ & $48(100,0)$ & \\
\hline Fundamental completo ou mais & $9(81,8)$ & $2(18,2)$ & II $(100,0)$ & \\
\hline \multicolumn{4}{|l|}{ Renda familiar (salário mínimo) } & 0,815 \\
\hline Inferior ou igual a I salário & $9(40,9)$ & $13(59,1)$ & $22(100,0)$ & \\
\hline
\end{tabular}




\begin{tabular}{l|c|c|c|c} 
& \multicolumn{3}{|c|}{ Frequência } & P \\
\hline & Ativas & Inativas & Total & \\
\hline Superior a I ou 2 salários & $\mathrm{N}(\%)$ & $\mathrm{N}(\%)$ & $\mathrm{N}(\%)$ & \\
\hline Quarto compartilhado & $14(37,8)$ & $23(62,2)$ & $37(100,0)$ & \\
\hline Não compartilha/compartilha com marido & $23(46,9)$ & $26(53,1)$ & $49(100,0)$ & \\
\hline Parentes & - & $10(100,0)$ & $10(100,0)$ & \\
\hline Total & $23(39,0)$ & $36(61,0)$ & $59(100,0)$ \\
\hline
\end{tabular}

Fonte: elaborada pelas autoras, 2012.

$\mathrm{Na}$ Figura I, estão descritas as principais causas encontradas para justificar a inatividade sexual na terceira idade das mulheres entrevistadas. A principal causa é a falta de parceiro, representando $25 \%$ do total, seguida de falta de privacidade (19,5\%), falta de vontade da mulher $(16,7 \%)$, vergonha do corpo $(13,9) \%$, dispareunia $(8,3 \%)$ e, com igual porcentagem, a falta de vontade do marido acreditar que têm idade para continuar a atividade sexual.

Figura I - Autorrelato das causas da inatividade sexual de mulheres idosas do CRAS do município de Aracaju (SE) em 2012 Causas da Inatividade Sexual na Terceira Idade, Aracaju (20I2)

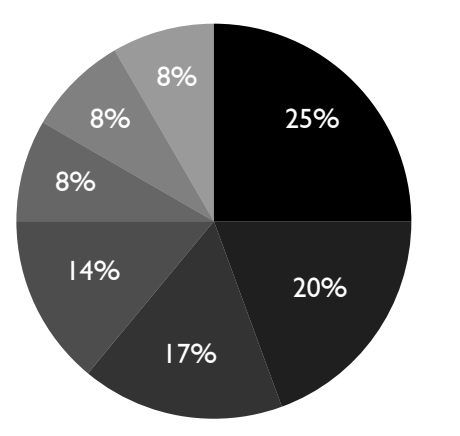

Ausência de parceiro
Ausência de privacidade
Ausência de vontade da mulher
Vergonha do corpo
Dispareunia
Ausência de vontade do parceiro

Acredita não ter mais idade para sexo

Fonte: elaborada pelas autoras, 2012.

Segundo relato das idosas, a principal causa da continuidade da atividade sexual na terceira idade é o fato de as mulheres ainda sentirem prazer, o que corresponde a $86,95 \%$, enquanto $13,04 \%$ têm relações sexuais por obri- gações matrimoniais. Quanto à frequência sexual, 60,87\% praticam uma vez por semana, 13,04\%, uma vez a cada duas semanas e $21,73 \%$, uma vez por mês (Figura 2).

Figura 2 - Assiduidade sexual de mulheres idosas do CRAS do município de Aracaju (SE) em 2012

Assiduidade do Sexo na Terceira Idade, Aracaju (2012)

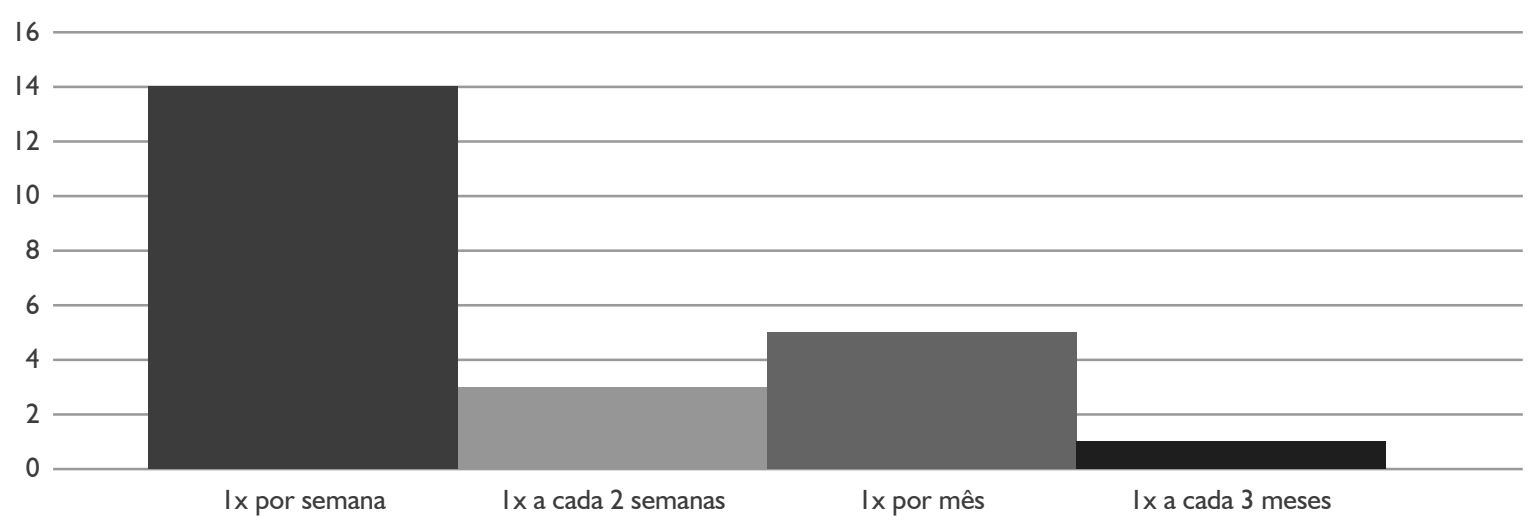

Fonte: elaborada pelas autoras, 2012. 
Quanto à presença de libido, $66,10 \%$ das mulheres referem que está presente e $33,89 \%$, não ter mais libido. Quanto às causas da ausência de libido, 8,3\% das mulheres referiram dispareunia, $12,5 \%$ terem vergonha do corpo e, a maioria, 79,16\%, referiu outros motivos. Em relação à inatividade sexual, 7I,42\% cessaram a prática sexual após 60 anos de idade, enquanto $28,57 \%$ cessaram antes dos 60 anos.

Sobre as mudanças fisiológicas sexuais provenientes do envelhecimento, $53 \%$ das idosas perceberam mudanças e $47 \%$ delas não notaram nenhuma diferença. Sobre a prevalência das alterações fisiológicas ocorridas no corpo das mulheres idosas citadas por elas durante a entrevista: $24,83 \%$ referiram mamas mais pêndulas; $19,60 \%$, atrofia vaginal; $17,64 \%$, ressecamento vaginal; $17 \%$, a diminuição da vontade de ter relações sexuais.

Com relação ao interesse das idosas em relacionar-se amorosamente após ficar viúva ou se divorciar (Figura 3), 45,5\% gostariam de se relacionar novamente e $54,5 \%$, não querem outro relacionamento. Em relação à opinião dos filhos sobre novos relacionamentos, $32,2 \%$ referiram que acreditam que seus filhos são a favor de que elas se relacionem novamente. A relação entre as duas variáveis foi estatisticamente significativa $(p=0,003)$.

Figura 3 - Percepção de mulheres idosas em relação a novas experiências sexuais e à aceitação dos filhos das idosas do CRAS do município de Aracaju (SE) em 2012

\section{Aberta para Nova Relação Afetiva}

Percepção de opnião negativa dos filhos

Percepção de opnião positiva dos filhos

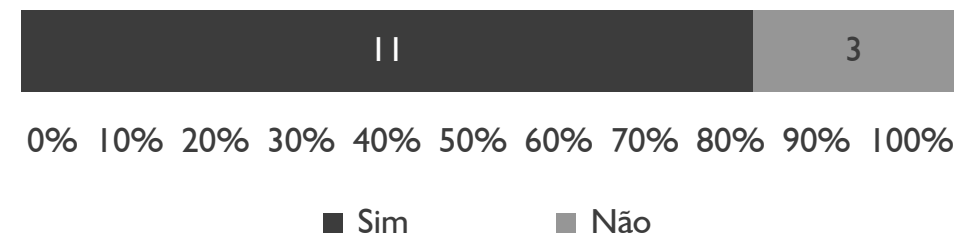

Fonte: elaborada pelas autoras, 2012.

\section{Discussão}

Os resultados apontaram que, na comunidade pesquisada, houve uma predominância de idosas na faixa etária de 60 a 70 anos de idade, sendo principalmente viúvas, que, na sua maioria, não tem interesse em um novo relacionamento. Quanto ao perfil socioeconômico, apresentam baixo nível de escolaridade e renda mensal variando entre um e dois salários mínimos.

Em relação ao perfil sociodemográfico encontrado, pôde-se observar como fatores relevantes, que não houve nenhuma mulher solteira dentre as entrevistadas e que a maioria era viúva. Isso se deve ao fato de a longevidade feminina ser maior do que a masculina, podendo estar associado a fatores genéticos, metabólicos e até mesmo pela maior preocupação com a prevenção em saúde (LEITE; OTTONI, 20I7; TOWER, 20I7).

Esse dado pode ser confirmado por Fernandes e demais autores (2019), que afirmam que as mulheres vivem mais do que os homens. Isso se atribui à maior disposição que elas têm para cuidar de si mesmas e buscar assistência. Portanto, estas são as mais plausíveis de ficarem viúvas e sozinhas. Sabendo que há uma forte tendência dos homens mais velhos se unirem a pessoas mais jovens.
A escolaridade foi associada significativamente com a atividade sexual. $O$ baixo grau de escolaridade pode vir a influenciar um melhor entendimento do idoso quanto às mudanças fisiológicas ocorridas durante o processo do envelhecimento, principalmente relacionado à menopausa e acerca de orientações de educação sexual que possa vir a ocorrer nas equipes de saúde da família (CARNEIRO et al., 2016).

Levando-se em conta os resultados obtidos, percebe-se que o nível socioeconômico dessas idosas é baixo, já que a maioria das entrevistadas vive com renda entre um e dois salários mínimos e muitas delas moram com filhos ou netos. Quanto à divisão de cômodos, obteve-se uma pequena porcentagem de idosas que divide o quarto com parentes, fato que foi associado significativamente com a atividade sexual.

O fato de ser viúva esteve associado à inatividade sexual. Segundo Mesquita Filho e demais autores (2018), as mulheres em geral sempre foram vítimas de preconceitos sexistas, sendo inferiorizadas em relação ao sexo oposto. Os homens quando viúvos geralmente voltam a se casar, mesmo em idade avançada, fato que não é comum entre as mulheres, que optam por viverem uma 
viuvez solitária e quase sempre assumem responsabilidades sobre toda a família.

Quanto aos fatores citados como motivo da inatividade sexual, destacam-se a falta de parceiro e falta de privacidade. Entende-se que para continuar exercendo a atividade sexual é necessário confiança do parceiro, privacidade e condições psicológicas favoráveis. Rodrigues e demais autores (2018) e Ferreira e demais autores (2015) apontaram alguns fatores que podem dificultar ou mesmo inibir o exercício da sexualidade entre os idosos como: a saúde física, os preconceitos sociais, a autoestima, conhecimento sobre sexualidade, status conjugal e a perda de privacidade, como, por exemplo, viver com os filhos.

Dentre as ativas sexualmente, a frequência da prática sexual é de uma vez por semana pelo motivo de sentirem prazer. Estima-se que aproximadamente $20 \%$ das mulheres acima de 60 anos são sexualmente ativas. A atividade sexual é limitada pela ausência de um parceiro disponível. Em alguns casos, o impulso sexual não diminui à medida que as mulheres envelhecem, e algumas relatam um aumento no impulso sexual (SANTOS et al., 2019).

Vale ressaltar que o organismo humano, com o passar dos anos, modifica-se por inteiro. A força muscular de todo o corpo diminui juntamente com o vigor físico, e os tecidos perdem a elasticidade. Essas alterações têm uma importância para a fisiologia da sexualidade. É necessário evitar uma comparação inadequada com a vida sexual de outras faixas etárias, pois a vida sexual continua existindo até $o$ fim da vida, sendo característico a cada sujeito que envelhece a frequência dos desejos e os ritmos das relações (MARCH, 20I8; VIEIRA; COUTINHO; SARAIVA, 2016).

Pode-se notar que, apesar do grande quantitativo de mulheres que não percebe as mudanças fisiológicas sexuais, quando feito outro questionamento onde foram colocadas as opções dessas mudanças, elas souberam expressar as ocorridas em seu corpo. Isso pode ser justificado pela falta de esclarecimento a respeito das alterações fisiológicas que ocorrem durante o processo de envelhecimento do ser humano. O que confirma que elas não receberam a devida educação sexual durante a vida.

A maioria das mulheres não apresentou nenhum sintoma especial, sendo os mais comuns as ondas de calor, cefaleia, tendência ao cansaço, instabilidade emocional e irritabilidade, que não são inevitáveis, porém podem ser aliviados ou resolvidos totalmente através de terapias de reposição hormonal (SANTOS; FAUSTINO, 20I8).

Pode-se notar que as porcentagens entre perceber e não perceber as mudanças são muito próximas. Portanto, as modificações ocorridas com o envelhecimento poderiam ser mais perceptíveis se houvesse um maior esclarecimento quanto a educação sexual no intuito de preparar a mulher para a menopausa e suas consequências.
Torna-se crucial lembrar que a atividade sexual é um fator determinante na qualidade de vida das pessoas por fazer parte das necessidades humanas básicas. Por isso, a informação tem papel relevante na sociedade, na família e na mídia que é o principal meio de comunicação. Os fatores psicossociais parecem ser os maiores inibidores do comportamento sexual na terceira idade, entre eles a falta de informação, a falta de parceiros, e comportamentos limitados, restringindo a vida dessas mulheres na sua relação com a sociedade. A mulher mais velha tem maiores dificuldades de reconstruir uma vida afetiva sexual pelo fator histórico e culturalmente ter sido educada de forma muito rígida (CABRAL et al., 2019).

Interpreta-se que, mesmo com o avançar da idade, muitas mulheres permanecem ativas e interessadas pelo sexo. A alta assiduidade sexual desfaz a visão preconceituosa de que idoso seja assexuado e prova que durante a velhice o desejo sexual pode diminuir, entretanto, o desejo e a necessidade de afeto permanecem. Por outro lado, uma minoria refere não ter desejo sexual, apresentando como motivo a cessação da vontade após terem enviuvado. Outras, por não confiarem nos homens e por acreditarem que eles não querem levar um relacionamento a sério, podendo lhes transmitir doenças.

Segundo Queiroz e demais autores (2015), intimidade implica confiança, credibilidade no parceiro, entregar-se sem medo, seguir em frente sem arrependimento, sendo o que é, e estar em contato com a realidade do outro, sem julgamentos. A manifestação da sexualidade nas idosas está diretamente ligada às experiências anteriores e ao modo de percepção das modificações biopsicossociais provenientes do envelhecimento. Assim, entende-se a importância da aceitação das alterações pela idosa, permitindo-se novas vivências (RODRIGUES; GONÇALVES, 2019). Conforme Souza e demais autores (2019), muitas mulheres utilizam essa etapa da vida como desculpa para se livrarem da obrigação de manterem uma vida sexual ativa, atribuindo a terceira idade à incapacidade sexual, evitando totalmente algo que antes já era indesejado.

Por fim, a crença de não aceitação dos filhos esteve associada à não estar aberta a novos relacionamentos. As idosas sofrem as consequências de seu envelhecimento biológico, tendo de conviver com as perdas típicas dessa fase e também com o comportamento preconceituoso sobre o relacionamento afetivo, amoroso e sexual, emitido pela sociedade, que pode ser um estímulo discriminativo que leva a extinção do comportamento sexual das idosas (BELL et al., 20I7).

\section{Conclusões}

Diante do estudo, constatou-se que a idade não interferiu nas relações afetivas e sexuais das idosas entrevistadas, mas outros fatores sociais, como escolaridade, 
estado civil e privacidade no quarto. $A$ idade não teve associação significativa com a atividade sexual e a maioria das idosas sexualmente ativas mantem relações sexuais por prazer, o que comprova que o aumento da idade não está relacionado com a diminuição do desejo sexual.

Muitas das mulheres viúvas e divorciadas têm receio de um novo relacionamento associado à percepção de que os filhos não aceitariam o envolvimento afetivo delas com um novo parceiro. Um dos desafios que as idosas enfrentam são os mitos e os preconceitos relacionados ao envelhecimento e à sexualidade, que acabam impedindo-as de desfrutá-la.

A equipe de saúde da família pode desempenhar um importante papel na quebra dos tabus relacionados à sexualidade na terceira idade. Por ter contato direto e vínculos com os indivíduos do território adscrito, é possível orientar a população em qualquer fase do ciclo vital quanto aos benefícios de uma vida sexual ativa e as disfunções sexuais decorrentes do envelhecimento. É necessário chamar a atenção dos órgãos públicos para desenvolver entre as equipes de saúde da família a educação sexual nos usuários atendidos, explicando o verdadeiro conceito da sexualidade que não se restringe somente ao ato sexual, a fim de acabar com os preconceitos na sociedade e preparar futuros idosos para uma senescência mais saudável. Bem como entendermos a sexualidade como algo essencial na vida de qualquer ser humano, que só traz benefícios para a saúde, bem-estar e satisfação.

\section{Referências}

ARAÚJO, B. J. et al. Qualidade de vida e sexualidade na população da terceira idade de um centro de convivência. Revista de Divulgação Científica Sena Aires, v. 6, n. 2, p. 85-94, 2017. Disponível em: http://revistafacesa.senaaires. com.br/index.php/revisa/article/view/282

ARAÚJO, S. L.; ZAZULA, R. Sexualidade na terceira idade e terapia comportamental: revisão integrativa. Revista Brasileira de Ciências do Envelhecimento Humano, v. 12, n. 2, p. I72-182, 2015. Disponível em: http://seer.upf.br/index. $\mathrm{php} / \mathrm{rbceh} /$ article/view/5054

\section{BELL, S. et al. Sexual Activity After 60: A} Systematic Review of Associated Factors. Sexual Medicine Reviews, v. 5, n. I, p. 52-80, 2017. Disponível em: https://www.smr.jsexmed.org/ article/S2050-052 I ( I6)00080-9/pdf

BRASIL. Ministério da Saúde. Secretaria de Atenção Básica. Departamento de Atenção Básica. Saúde sexual e saúde reprodutiva. Brasília, DF: Ministério da Saúde, 20I3.
CABRAL, N. E. S. et al. Understanding sexuality by rural elderly women. Revista Brasileira de Enfermagem, v. 72, p. 147-I52, 2019. Supl. Disponível em: https://www.scielo.br/pdf/reben/ v72s2/pt_0034-7 I67-reben-72-s2-0 I47.pdf

CAMPOS, A. C. V.; FERREIRA, E. F.; VARGAS, A. $M$. D. Determinantes do envelhecimento ativo segundo a qualidade de vida e gênero. Ciência \& Saúde Coletiva, v. 20, n. 7, p. 222I-2237, 2015. Disponível em: https://www.scielo.br/pdf/csc/ v20n7/|4| 3-8| 23-csc-20-07-222I.pdf

CARNEIRO, J. A. et al. Prevalence and factors associated with frailty in non-institutionalized older adults. Revista Brasileira de Enfermagem, v. 69, n. 3, p. 408-415, 2016. Disponível em: https://www.scielo.br/pdf/reben/v69n3/00347167-reben-69-03-0435.pdf

EVANGELISTA, A. R. et al. Sexuality in old age: knowledge/attitude of nurses of Family Health Strategy. Revista da Escola de Enfermagem da USP, v. 53, p. I-8, 2019. Disponível em: https://www. scielo.br/pdf/reeusp/v53/I 980-220X-reeusp53-e03482.pdf

FERNANDES, D. S. et al. Avaliação da capacidade funcional de idosos longevos amazônidas. Revista Brasileira de Enfermagem, v. 72, p. 49-55, 2019. Supl. 2. Disponível em: https://www.scielo.br/pdf/ reben/v72s2/pt_0034-7167-reben-72-s2-0049.pdf

FERREIRA, S. M. A. et al. Barreiras na inclusão da sexualidade no cuidado de enfermagem de mulheres com câncer ginecológico e mamário: perspectiva das profissionais. Revista LatinoAmericana de Enfermagem, v. 23, n. I, p. 82-89, 2015. Disponível em: https://www.scielo.br/pdf/ rlae/v23n I/pt_0 I04-I I69-rlae-23-0 I-00082.pdf

FREIRE, A. K. S. et al. Aspectos psicossociais da sexualidade na adolescência: diálogos e aprendizagem na escola. Semina: Ciências Biológicas e da Saúde, v. 38, n. I, p. 3-I4, 2017. Disponível em: http://www.uel.br/revistas/uel/ index.php/seminabio/article/view/26736

LEITE, M. E.; OTTONI, M. A. M. Análise espacial e acessibilidade dos idosos nos centros de referência de assistência social em um município de Minas Gerais. O Social em Questão, v. 20, n. 38, p. 269-88, 2017. Disponível em: http:// osocialemquestao.ser.puc-rio.br/media/OSQ_38_ SL_art_2_Leite_Ottoni.pdf 
MARCH, A. L. Sexuality and intimacy in the older adult woman. Nursing Clinics, v. 53, n. 2, p. 279287, 2018. Disponível em: https://www.nursing. theclinics.com/article/S0029-6465(I8)30007-0/ abstract

MESQUITA FILHO, M. et al. O preconceito contra a mulher entre trabalhadores da Atenção Primária em Saúde. Ciência \& Saúde Coletiva, Rio de Janeiro, v. 23, p. 349I-3504, 20 I8. Disponível em: https://www.scielo.br/pdf/csc/v23n I I/ I4 I38I23-csc-23-II-349I.pdf

QUEIROZ, M. A. C. et al. Representações sociais da sexualidade entre idosos. Revista Brasileira de Enfermagem, v. 68, n. 4, p. 662-667, 2015. Disponível em: https://www.scielo.br/pdf/reben/ v68n4/0034-7I 67-reben-68-04-0662.pdf

RODRIGUES, D. M. M. R. et al. The dialogic educational pathway as a strategy of care with elderly women in sexuality. Escola Anna Nery, v. 22, n. 3, 2018. Disponível em: https://www. scielo.br/pdf/ean/v22n3/pt_|4|4-8|45-ean-2 I779465-EAN-20 I7-0388.pdf

RODRIGUES, W. P.; GONÇALVES, P. D. Envelhecimento: qualidade de vida e bem-estar das mulheres idosas. Scire Salutis, v. 9, n. I, p. 30-36, 2019. Disponível em: https://sustenere. co/index.php/sciresalutis/article/view/CBPC22369600.2019 .001 .0004

SANTOS, A. D. et al. Concepção de mulheres idosas sobre sexualidade na velhice. Revista de Enfermagem UFPE on line, Recife, v. I3, p. I-8, 20 I9. Disponível em: https://periodicos. ufpe.br/revistas/revistaenfermagem/article/ view/24I 752/33974

SANTOS, D. L. R.; FAUSTINO, A. M. Saúde sexual e sexualidade de mulheres idosas: revisão de literatura. Revista Gestão e Saúde, v. I, n. 3, p. 674-9I, 2017. Num. Esp. Disponível em: https://periodicos.unb.br/index.php/rgs/article/ view/I0423

SANTOS, N. F. V. et al. Ações de Educação em Saúde Sobre Sexualidade com Idosos. Saúde em Redes, v. 3, n. 2, p. 162-I7I, 20I7. Disponível em: http://revista.redeunida.org.br/ojs/index.php/redeunida/article/view/828
SOUSA, N. F. S. et al. Active aging: prevalence and gender and age differences in a populationbased study. Cadernos de Saúde Pública, v. 34, n. I I, p. I-16, 20I9. Disponível em: https://www. scielo.br/pdf/csp/v34n I I//678-4464-csp-34II-e00I733I7.pdf

SOUZA, C. L. et al. Aging, sexuality and nursing care: the elderly woman's look. Revista Brasileira de Enfermagem, v. 72, p. 7I-78, 2019. Disponível em: https://www.scielo.br/pdf/reben/v72s2/ pt_0034-7I67-reben-72-s2-007I.pdf

SOUZA, M. P. Apercepção dos idosos sobre a sexualidade: revisão sistemática da literatura. Saúde \& Transformação Social, v. 6, n. I, p. |24-|3|, 20I5. Disponível em: http:// incubadora.periodicos.ufsc.br/index.php/ saudeetransformacao/article/view/2703

TOWER, J. Sex-specific gene expression and life span regulation. Trends in Endocrinology \& Metabolism, v. 28, n. I0, p. 735-747, 2017. Disponível em: https://doi.org/10.1016/j. tem.2017.07.002

UCHÔA, Y. S. et al. Sexuality through the eyes of the elderly. Revista Brasileira de Geriatria e Gerontologia, v. 19, n. 6, p. 939-949, 2016. Disponível em: https://www.scielo.br/pdf/rbgg/ v19n6/pt_1809-9823-rbgg-19-06-00939.pdf

VIEIRA, K. F. L.; COUTINHO, M. P. L.; SARAIVA, E. R. A. A sexualidade na velhice: representações sociais de idosos frequentadores de um grupo de convivência. Psicologia Ciência e Profissão, v. 36, n. I, p. 196-209, jan./mar. 2016. Disponível em: https://www.scielo.br/pdf/pcp/v36n I/I 982-3703pcp-36-I-0196.pdf

\section{WORLD HEALTH ORGANIZATION.}

Envelhecimento ativo: uma política de saúde. Brasília, DF: Organização Pan-Americana da Saúde, 2005.

WORLD HEALTH ORGANIZATION. Sexual and reproductive health. 2006. Disponível em: https:// www.who.int/reproductivehealth/topics/sexual_ health/sh_definitions/en/. Acesso em: 29 maio 2020. 